Methods The study was conducted by members of CHARGE (Child Health Applied Research Group: East-midlands) using a methodology informed by The PRISMA Statement (Preferred Reporting Items for Systematic reviews and Meta-Analysis).

A clinical question using a standard PICO (Patient, Intervention, Comparison, Outcome) format was used to inform the search terms and search strategy. The search was executed on Medline, Embase, The Cochrane Library, two meta-search engines and ISI-Web of Science. In addition, a search of the grey literature was conducted. Retrieved studies were independently appraised by two reviewers for relevance and quality using the Cochrane Collaboration's tool for assessing risk of bias. Data was extracted onto a standardised proforma.

Results Only one small clinical trial $(\mathrm{n}=39)$ could be included in our final analysis and this showed statistically non-significant effects for parentally reported improved behaviour (58\% [drug] vs. $53 \%$ [placebo]). There was no difference between the two groups for adverse outcomes.

This trial and two excluded trials highlighted the critical importance of proper methodology for conducting future trials in autism. These include the need for appropriate: power, outcomes and follow-up, and due consideration of the clinical spectrum of autistic patients involved in the trials

Conclusion There is insufficient evidence to support the use of dimethylglycine for the treatment of children with autism. Further robust research is required on this topic

\section{DIGEORGE SYNDROME: COGNITIVE AND BEHAVIOURAL DEVELOPMENT FROM BIRTH TO ADOLESCENCE}

doi:10.1136/archdischild-2012-302724.0634

'M Bua, 'E Sgaravatti, 'M Blazina, 'F Menegazzo, ${ }^{1} \mathrm{P}$ Facchin, ${ }^{2} \mathrm{E}$ Ruga. ${ }^{1}$ Rare Diseases Registry, Paediatrics Department, University of Padova, Italy; '2Paediatrics Department, University of Padova, Padova, Italy

Background and Aims DiGeorge syndrome (DGS) is a rare disease associated with a microdeletion of chromosome 22q11.2. Among clinical signs: heart defects, immunological alterations, psychiatric disorders. DGS children present developmental delay. The aim of this study is to assess cognitive and behavioural development of DGS paediatric patients.

Methods Cognitive profile was assessed in a prospective cohort of DGS children referred to Paediatrics Department-Padua University (1993-2012). For a sample of 20 children (11 females, 9 males; $25 \%$ $<2$ yrs, $25 \% 3-5$ yrs, $50 \%>6 \mathrm{yrs}$ ), informations were collected on diagnosis, surgical interventions, hospitalizations, treatments/ rehab training programs. Cognitive profile was assessed using Griffith's Mental Development Scales (GMDS) and Wechsler Intelligence Scale for Children-III (WISC-III), depending on children's age. Behavioural profile was assessed using Child Behavior Checklist (CBCL). Univariate and multivariate descriptive analyses were performed.

Results For younger children (GMDS, 10 children), global mental development resulted: $15.4 \%$ moderate retardation, $61.5 \%$ mild retardation, $30.8 \%$ borderline, $7.7 \%$ low normal, $15.4 \%$ normal. Worse scores are observed in the subscales: language, performance, eye-hand coordination and practical reasoning. For older children (WISC-III, 10 children), 76.9\% had Mental Retardation (15.4\% moderate MR, $61.5 \%$ mild MR), and $23.1 \%$ got low Global IQ scores (7.7\% borderline, $15.4 \%$ low normal). Behavioural profile is barely normal in pre-school children and becomes borderline/clinical in school children (100\% disadaptative functioning, $40 \%$ internalizing problems, $20 \%$ externalizing problems).

Conclusions DGS patients have a wide spectrum of developmental delays, which require tailor-made rehab programs, and a worsening in behavioural profile in pre-adolescence and adolescence.

\section{DEFICITS OF MOTION PERCEPTION IN CHILDREN WITH TREATED CONGENITAL HYPOTHYROIDISM (CI)}

doi:10.1136/archdischild-2012-302724.0635

F Tinelli, G Purpura, G Cioni, S Bargagna. Department of Developmental Neuroscience, IRCCS-Stella Maris Institute, Calambrone, Italy

Background and Aims Thyroid hormones have an important role throughout prenatal and postnatal nervous system development. They are involved in several processes such as neurogenesis, gliogenesis, myelination, as shown in many cases of deficiency like CI Some could be reversed after adequate supplementation of thyroid hormones at birth, however there are other cellular processes highly sensitive to low levels of thyroid hormones and lasting a limited period of time during which if thyroid hormone action is lacking or deficient, the functional and structural damages would produce permanent defects. Visual system is particular vulnerable to thyroid hormones and for this reason we decided to study children with CI early treated by means of visual motion tasks to evaluate basic and high level functions.

Methods and results: Ten children with early treated CI and ten controls matched for sex and age and Intelligence Quotient were enrolled in the study. We found no differences in basic visual functions. Motion perception was assessed by two alternative forced choise of direction of motion for different levels of noise corruption displayed on the screen for a limited lifetime. Three types of coherent motion were studied: rotational, radial and translational motion. We found a statistical difference in the thresholds for radial motion.

Conclusions Our results seem to confirm that CI can influence the development of the visual dorsal stream, a pathway particularly vulnerable during the last three months of intrauterine life.

\section{BIPOLAR DISORDER AND PREGNANCY - NEURODEVELOPMENTAL OUTCOME OF CHILDREN EXPOSED TO MATERNAL ILLNESS WITH OR WITHOUT LITHIUM DURING PREGNANCY}

doi:10.1136/archdischild-2012-302724.0636

1,2L Forsberg, ${ }^{2} \mathrm{G}$ Berglund, ${ }^{1,3} \mathrm{~L}$ Naverr, ${ }^{4} \mathrm{M}$ Ljungdahl, ${ }^{4,5} \mathrm{M}$ Adler, ${ }^{1,2} \mathrm{~K}$ Wide. ${ }^{1} \mathrm{Dep}$ of Clinical Science, Intervention and Technology, Karolinska Institutet; ${ }^{2}$ Dep of Pediatric Neurology; ${ }^{3}$ Dep of Neonatology, Astrid Lindgrens Pediatric Hospital, Karolinska University Hospital Huddinge; ${ }^{4} \mathrm{Dep}$ of Psychiatry Southwest Stockholm, SLSO; ${ }^{5} \mathrm{Dep}$ of Clinical Neuroscience, Karolinska Institutet, Stockholm, Sweden

Background and Aims Bipolar disorder (BD) is a chronic psychiatric condition. Lithium is the most common mood stabilizing drug during pregnancy. It is unknown whether lithium exposure in utero may have negative effects on neurodevelopment. This study aims to investigate the health and cognitive development of preschool children born to mothers with BD.

Methods 10 women with BD who had given birth 2006 or 2007 were recruited from a psychiatric center. 4 of them had been medicating with lithium during pregnancy. 4 women without psychiatric illness were included. At age $4-5$ the children were tested by a child psychologist, using Wechsler Preschool and Primary Scale of Intelligence (WPPSI) and physically examined. The mental health and social situation of the mother was assessed by a psychiatric nurse or psychiatrist.

Results WPPSI results were available for 9 children. Mean ful scale IO of children exposed to lithium during pregnancy $(n=3)$ was 108 and for unexposed children $(n=6)$ 111, no significant difference. One child, born to a mother with bipolar disease but not exposed to lithium, was born prematurely and tested in the lower normal range of the IQ scale. One child, not exposed to lithium, had been diagnosed with ADHD. All children were in good general health. 